\begin{tabular}{|l|l|l|}
\hline Revista & & $\begin{array}{l}\text { Revista Latina de Sociología (RELASO) } \\
\text { Vol. 9, núm. 2 (2019), pp. 59-76 } \\
\text { ISSN-e 2253-6469 } \\
\text { DoI: https://doi.org/10.17979/relaso.2019.9.2.6827 }\end{array}$ \\
de Sociologia & &
\end{tabular}

\title{
Aplicação do respondent driven sampling (rds) em adolescentes em situação de rua
}

\section{Application of respondent-driven sampling (RDS) among street teenagers}

Aplicación de muestra dirigida por el entrevistado (dem) a los adolescentes en la situación de la calle

Givanildo da Silva Nery

Universidade Federal da Bahia givanildogsn@hotmail.com

ORCID: https://orcid.org/0000-0001-9004-8689

Rosely Cabral de Carvalho

Universidade Estadual de Feira de Santana roselycarvalho056@gmail.com

ORCID: https://orcid.org/0000-0002-1060-2780

Sinara de Lima Souza

Universidade Estadual de Feira de Santana sinarals@uefs.br

ORCID: https://orcid.org/0000-0001-8003-2093

Luzimara Gomes Melo

Universidade Estadual de Feira de Santana luzimara.gm@gmail.com

ORCID: https://orcid.org/0000-0002-7832-860x

José Eduardo Ferreira Santos

Universidade Católica do Salvador ferreirasantosenator@gmail.com

ORCID: https://orcid.org/0000-0003-2949-6022 


\begin{tabular}{|l|l|l|}
\hline Revista & $\begin{array}{l}\text { Revista Latina de Sociología (RELASO) } \\
\text { Vol. 9, núm. 2 (2019), pp. 59-76 } \\
\text { ISSN-e 2253-6469 } \\
\text { DoI: https:// doi.org/10.17979/relaso.2019.9.2.6827 }\end{array}$ \\
de Sociologia &
\end{tabular}

\title{
Resumo
}

Relato da experiência do uso da metodologia Respondent - Driven Sampling (RDS), em pesquisa com 86 adolescentes em situação de rua em uma cidade da Bahia. Objetivo: descrever as principais etapas de implementação do método, seus limites e possibilidades. Para a coleta de dados foi realizado mapeamento geográfico (feiras livres, sinaleiras, rodoviária), utilizandose de ondas de recrutamento e identificação das sementes de sujeitos dentro da população-alvo. Verificou-se que o reconhecimento da pluralidade de modos de vida, as características dos territórios pesquisados e as vivências itinerantes dos adolescentes em situação de rua influenciam na determinação dos limites e possibilidades de uso da técnica. A dinâmica social e a mobilidade ativa dos adolescentes são características que representam desafios na replicação do estudo com a mesma população e território.

Palavras-chave: técnica de pesquisa; risco psicossocial; vulnerabilidade social, adolescência, situação de rua

\begin{abstract}
This article reports on the experience of using a Respondent-Driven Sampling (RDS) methodology to survey a group of 86 street teenagers in a city in Bahia (Brazil). The purpose of the study is to explain how the methodology was implemented, together with the limitations and possibilities of RDS as a sampling technique. The data were collected using geographical mapping (open markets, traffic lights, roads), recruitment waves and seed identification of individuals within the target population. The usefulness and limitations of the technique were found to be influenced by the diverse reality of street teenagers' way of life and the contexts in which they live, as well as the nomadic nature of their experiences. The social dynamics between the teenagers and their active mobility represent an obstacle to replicating the study with the same population and territory.
\end{abstract}

Keywords: Research technique; psychosocial risk; social vulnerability; teenagers; street living.

\section{Resumen}

Informe sobre la experiencia del uso de la metodología de Muestreo conducido por encuestados (RDS), en una encuesta a 86 adolescentes de la calle en una ciudad de Bahía. Objetivo: describir las principales etapas de implementación del método, sus límites y posibilidades. Para la recopilación de datos, se realizó un mapeo geográfico (mercados abiertos, semáforos, carreteras), utilizando ondas de reclutamiento e identificación de las semillas de los sujetos dentro de la población objetivo. Se encontró que el reconocimiento de la pluralidad de formas de vida, las características de los territorios investigados y las experiencias itinerantes de los adolescentes en la calle influyen en la determinación 


\begin{tabular}{|l|l|l|}
\hline Revista & & $\begin{array}{l}\text { Revista Latina de Sociología (RELASO) } \\
\text { Vol. 9, núm. 2 (2019), pp. 59-76 } \\
\text { ISSN-e 2253-6469 } \\
\text { DoI: https:// doi.org/10.17979/relaso.2019.9.2.6827 }\end{array}$ \\
de Sociologia & &
\end{tabular}

de los límites y las posibilidades de utilizar la técnica. La dinámica social y la movilidad activa de los adolescentes son características que representan desafíos para replicar el estudio con la misma población y territorio.

Palabras clave: técnica de investigación; riesgo psicosocial; vulnerabilidad social, adolescencia, falta de vivienda

\section{Introdução}

As pessoas em situação de rua possuem uma multiplicidade de vulnerabilidades que estão relacionadas à própria precariedade de suas condições de vida, a reprodução dos ciclos de pobreza e aos efeitos da marginalização (Trino, Machado \& Rodrigues, 2015).

Existem, nesse sentido, táticas de sobrevivência na rua que são frequentemente utilizadas ou desenvolvidas por diferentes grupos que vivem nesses espaços, com a finalidade de garantir sua integridade, as quais incluem o tipo de lugar, mais ou menos seguro para dormir, a maneira como se relacionam com as pessoas ao seu redor (comerciantes, policiais e domiciliados), o tipo de trabalho produzido, a forma se alimentar e a postura forte e resistente perante os desafios do cotidiano (Kunz, Heckerls \& Carvalho, 2014).

Moura, Silva e Noto (2009) compreendem que o espaço da rua apropriado por crianças e adolescentes se trata não apenas de local de moradia e sobrevivência como também uma rede de existência social, a qual possibilita formas diversas e conflitantes de vida, se constituindo como uma cultura alternativa que auxilia no processo de adaptação social às realidades difíceis e antagônicas.

Ressalta-se que existe ainda na literatura uma lacuna de modelos teóricos e pesquisas empíricas relacionadas à realidade de vida de adolescentes em situação de rua e métodos e técnicas adequadas a sua compreensão, bem como os limites e potencialidades do trabalho com este público, os quais possam compreender e significar os processos de vulnerabilidade atravessado por essa população e acessar os fatores de proteção e risco que acometem tais sujeitos (Koller \& Hutz, 1997; Neiva-silva \& Koller, 2002).

Uma resposta a esse desafio é a técnica do Respondent Driven Sampling (RDS), enquanto método de pesquisa desenvolvido em 1997, cuja origem remonta inicialmente às diversas tentativas de inclusão de intervenções que fossem eficazes na prevenção ao HIV/AIDS (Heckathorn, 1997; Schonlau \& Liebau, 2012). Essa técnica possui respaldo teórico e metodológico em função de sua expressiva característica de ser um método de amostragem que busca preservar o rigor científico, mas ao mesmo tempo apresenta como limitações iniciais, as características semelhantes entre recrutados e recrutadores e a discrepância no tamanho das redes (National Alliance of State \& Territorial AIDS Directors, Nastad, 2014; Kendall, 2006).

As características do RDS como técnica de amostragem apresentam similaridade com outras ferramentas como snowball sampling (amostragem bola de neve), entretanto há uma diferenciação, na medida em que possibilita a cada entrevistado estimar o tamanho dessa população, de sua rede 


\begin{tabular}{|l|l|l|}
\hline Revista & & $\begin{array}{l}\text { Revista Latina de Sociología (RELASO) } \\
\text { Vol. 9, núm. 2 (2019), pp. 59-76 } \\
\text { ISSN-e 2253-6469 } \\
\text { DoI: https:// doi.org/10.17979/relaso.2019.9.2.6827 }\end{array}$ \\
de Sociologia & &
\end{tabular}

social, e ao mesmo tempo, o grau de semelhanças em diferentes momentos (ondas de recrutamento), convalidando tamanhos e heterogeneidades dos participantes do grupo entrevistado (Schonlau \& Liebau, 2012).

Nesse contexto, a proposta de relatar o processo de implementação e uso da técnica Respondent Driven Sampling (RDS) é importante a pesquisadores interessados em metodologias alternativas, para o desenvolvimento de estudos com populações de difícil acesso, além de contribuir para o monitoramento de possíveis erros e de enfoques diferenciados para essa abordagem.

Sobre a população em situação de rua, especificamente, os modos de organização e distribuição dos espaços urbanos e sociais na sua lógica de exclusão ou inclusão social privilegiam uns e negligenciam outros, no acesso aos serviços de saúde que não possui um elo fortalecido entre as políticas sociais e de saúde, dificultando seu alcance pelos diferentes pesquisadores e profissionais de saúde (Varanda \& Adorno, 2004).

Assim, o diálogo e a articulação teórico-metodológica dessas questões que, nessa pesquisa, chamou-se de "exclusão social", possibilita o entendimento do fenômeno como um processo de ruptura dos laços sociais, que segundo Nascimento (2009), tem características multidimensionais, geográficas e variadas (pobres, catadores de lixo, moradores de rua, usuários ou dependentes de drogas e sem-teto), os quais vivenciam situações de desfiliação com o trabalho, ruptura dos vínculos societários e portanto necessitam de uma abordagem metodológica e de pesquisa diferenciada.

Baseado nessas questões, esse artigo buscou responder através de um relato de experiência a seguinte questão: como a técnica Respondent Driven Sampling (RDS) se adequa a realidade de vulnerabilidade e risco vivenciada por adolescentes em situação de rua e quais os limites e possibilidades do uso de tal técnica? Concomitante a isso, o objetivo deste trabalho foi descrever as principais etapas de implementação da técnica e sua adequação à dinâmica de vida de adolescentes em situação de rua.

\section{Contextos Urbanos e Vulnerabilidade de Adolescentes em Situação de Rua}

As pesquisas com RDS têm sido aplicadas, tendo como objeto de estudo populações vulneráveis, buscando desvendar o papel das redes sociais, em ambientes de pessoas em situação de rua, contextos que são historicamente marcados por condições sociais que se vinculam a transmissão do HIV/AIDS, uso de drogas, violência e moradia incerta na rua (Escorel, 2009; Kunz, Heckerls, \& Carvalho, 2014).

A articulação teórico-metodológica da técnica RDS em contextos urbanos e de vulnerabilidade de adolescentes em situação de rua pode permitir conhecer a cultura e dinâmicas populacionais nesses espaços e, ao mesmo tempo, minimizar possíveis vieses de diferenças e similaridades em populações diversas, tais como homens que fazem sexo com homens, usuários de drogas, trabalhadores do sexo e populações em situação de rua (Carvalho et al, 2019).

Os modos de vida na rua e os espaços geográficos, socialmente construídos, desde a sua concepção, numa perspectiva inteiramente biográfica das narrativas elaboradas, são carregados de riscos que forçam deslocamentos contínuos e apropriação de ferramentas e artimanhas importantes, 


\section{Revista \\ Latina \\ de Sociologia}

Revista Latina de Sociología (RELASO)

Vol. 9, núm. 2 (2019), pp. 59-76

ISSN-e 2253-6469

DOI: https://doi.org/10.17979/relaso.2019.9.2.6827

na adaptação aos diversos tipos de ambientes com os quais é preciso a todo instante negociar, para assegurar a própria existência (Matias, 2011; Kunz, Heckerls \& Carvalho, 2014).

Schenker e Minayo (2005) ao discutir adolescência, comportamentos de riscos psicossociais e uso de drogas, aborda que existe um paradigma sistêmico, ou seja, um modelo que relaciona, intercruza e calcula fatores de risco e proteção associados ao estilo de vida de cada sujeito em processo de desenvolvimento.

Escorel (2009) ressalta que a condição de estar vivendo de rua tem uma influência direta na presença maior de riscos e problemas a saúde, uma vez que existe uma relação entre o tempo gasto na rua e os impactos sobre a condição de saúde. A autora revela em dados de pesquisa realizada com a população em situação de Rua no Brasil, que um terço das pessoas nessas condições há mais de cinco anos passam por algum processo de cronificação, grave adoecimento e precariedade das condições de vida.

Esses fatores podem ser considerados os que mais mitigam a situação de saúde e aumentam a probabilidade de adoecimento de pessoas em situação de rua, conforme destaca o Ministério do Desenvolvimento Social em levantamento nacional com esta população, onde 30\% dos entrevistados revelaram ter tido algum problema de saúde; dentre os mais citados são hipertensão $(10,1 \%)$, problemas psiquiátricos e mentais (6,1\%), diabetes (5,4\%) e HIV/AIDS (5,1\%) (Brasil, 2009).

Em mais recente censo sobre esta população, em 71 cidades brasileiras, foi revelado a existência de pelo menos 31.922 adultos em situação de rua (Brasil, 2019). Essa pesquisa apresenta os mais recorrentes motivos alegados pelas pessoas em situação de rua para se encontrarem em tal condição, quais sejam: os problemas com álcool/drogas (36\%), o desemprego (30\%) e por fim as desavenças com a familiares (29\%).

Nesse sentido, as vulnerabilidades sociais assumem a ideia de um o conjunto de eventos contextuais e ambientais, cujas características ameaçam a integridade biopsicossocial dos indivíduos, os quais necessitarão de atendimento especial e proteção do estado, da família e da sociedade, como forma de minimizar as situações de risco, tais como as enfermidades, violências, exploração, negligência ou quebra dos direitos humanos (Bittencourt, França \& Goldim, 2015; Adorno, 2011).

Revela-se, nesse ponto, a exclusão social que somada aos processos de exposição à diferentes riscos aos quais são expostos jovens e adolescentes no cenário das ruas, constatam que eles praticam a mendicância, são guardadores de carro, flanelinhas, pedintes, vendedores de doces e adesivos e utilizam de tais serviços, para auxiliar seus pais ou mesmo para o auto sustento nas ruas (Nascimento, 2009).

Nos contextos urbanos das vivências de crianças e adolescentes pobres, a exclusão, a vulnerabilidade, a violência e exploração andam sempre juntas, numa relação permeada pela fragmentação geográfica, pela má ordenação social dos espaços e práticas sociais discriminatórias (Mello, 2001).

Nesse sentido, intervenções adequadas a essas e outras problemáticas, no campo da saúde pública, devem estar refletidas nas políticas e ações voltadas à população em situação de rua. Desafio que envolve gestores e governantes, para a compreensão das trajetórias e contextos sociais que situam 


\begin{tabular}{|l|l|l|}
\hline Revista & & $\begin{array}{l}\text { Revista Latina de Sociología (RELASO) } \\
\text { Vol. 9, núm. 2 (2019), pp. 59-76 } \\
\text { ISSN-e 2253-6469 } \\
\text { DoI: https://doi.org/10.17979/relaso.2019.9.2.6827 }\end{array}$ \\
de Sociologia & &
\end{tabular}

pessoas em situação de rua de diferentes faixas etárias, com riscos e inúmeros prejuízos à saúde, como uso de drogas e HIV/AIDS. (Schwonke, Fonseca \& Gomes, 2009; Trino et al.; 2015).

\section{Revisão de Literatura: componentes teóricos e metodológicos}

Na literatura são limitadas as investigações cientificas que priorizam o desenvolvimento de métodos e técnicas de acesso a realidade de vida de adolescentes em situação de rua, assim a utilização de diversas metodologias e dados variados tanto sobre a população de rua quanto em relação ao uso de substâncias psicoativas é de fundamental importância para que se possa traçar políticas de cuidado e intervenções especificas, em grupos populacionais sob risco, e ao mesmo tempo elaborar metodologias adequadas para alcance dessas populações e que dialoguem com perspectivas investigativas de abordagens qualitativas, quantitativas e/ou multimétodos ( Koller \& Hutz, 1997, Morell, Silva, Lacerda \& Araújo, 2010).

Baseado nessa questão a metodologia Respondent Driven Sampling (RDS), apropriada a grupos que vivem sob extremo risco e vulnerabilidade social, se apresentam como grande contribuinte no acesso a populações vulneráveis e de difícil contato, tais como, e em especial, trabalhadores do sexo, podendo ser estendida a outros grupos como aqueles em situação de rua pelo qual estudamos (Morell et al, 2010).

Essa técnica, cuja tradução da língua inglesa “Respondent Driven Sampling” para o português compreende-se como "amostragem dirigida pelo participante”, se baseia num alistamento de pares e utilização destes para o recrutamento de novos indivíduos para a pesquisa com o intuito descobrir o tamanho das redes sociais estabelecidas entre esses indivíduos e através disso ter uma estimativa ponderada da população alvo (Pinho, 2010).

De acordo com Damacena, Szwarcwald e Barbosa-Júnior (2011), a utilização do RDS depende da realização de várias etapas, descritas na Figura 1; uma das primeiras e principais é a seleção, dentro da população alvo, do que se chama "sementes", ou seja, os indivíduos da população responsáveis pela eleição, dentro de seu ciclo de vínculos sociais, de um número fixo de pares adequado as suas características pessoais e aos critérios de inclusão do referido estudo.

Na revisão dos componentes teóricos e práticos do RDS destaca-se a perspectiva ideológica adotada por essa metodologia, a qual entende que as redes sociais possuem uma extensão e assumem um papel fundamental nas interações dos indivíduos, de modo que as mesmas possibilitam uma amostra final adequada às diversas propostas de pesquisa e/ou objeto de estudo (Morell et al, 2010). Entretanto vale salientar as limitações metodológicas, como por exemplo a complexidade de generalização dos dados; a dificuldade de assegurar a aleatoriedade uma vez que o estudo tem como foco redes de relações pelas quais os indivíduos recrutadores (sementes) mantêm uma prévia relação com aqueles que serão recrutados e a polêmica da questão dos incentivos, que apresenta visões variadas na literatura e abrindo margens para diversos questionamentos (Pinho,2010).

\section{Caracterização da área de estudo, público-alvo e técnica utilizada}




\begin{tabular}{|l|l|l|}
\hline Revista & $\begin{array}{l}\text { Revista Latina de Sociología (RELASO) } \\
\text { Vol. 9, núm. 2 (2019), pp. 59-76 } \\
\text { ISSN-e 2253-6469 } \\
\text { DoI: https://doi.org/10.17979/relaso.2019.9.2.6827 }\end{array}$ \\
\hline
\end{tabular}

Os dados desta pesquisa foram coletados no município de Feira de Santana, cidade do interior baiano, por meio de um levantamento com 86 adolescentes em situação de rua definindo diversas etapas interligadas entre si, aos pressupostos teóricos e metodológicos do RDS e a consecução dos objetivos do estudo. Tais etapas seguem descritas na (Figura 1) destacada em trabalho anterior (Nery, 2018; Carvalho et al, 2019).

Fig. 1. Etapas da Pesquisa

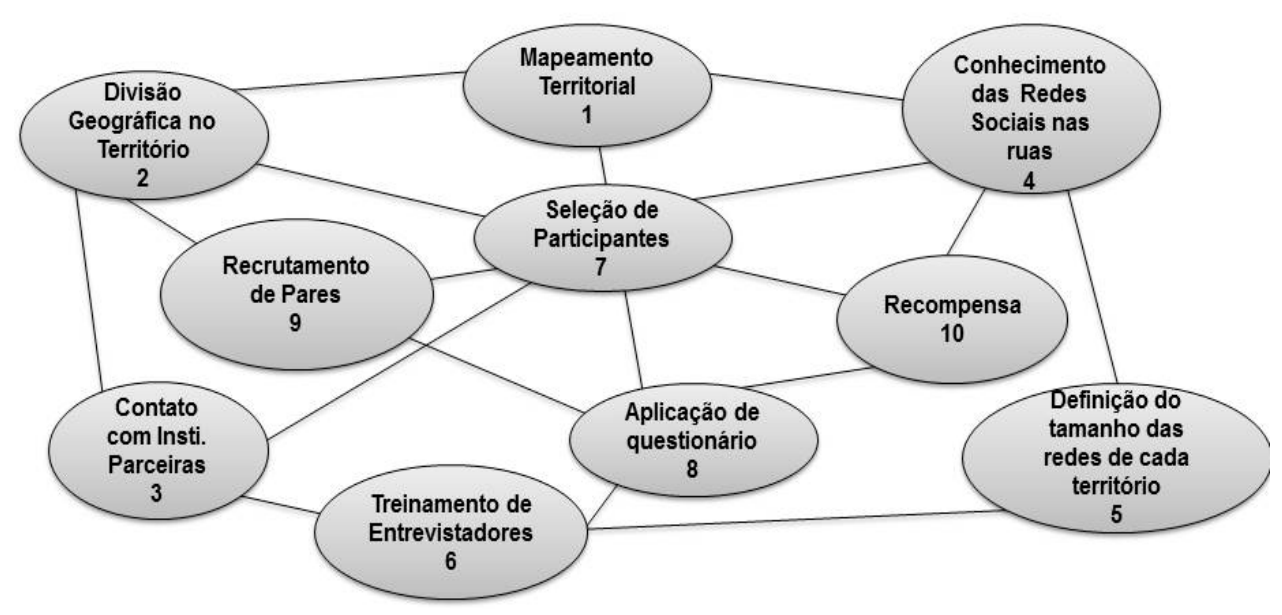

Fonte: Elaboração dos autores

No mapeamento das redes sociais de adolescentes em situação de rua e divisão geográfica do território, ocorreram reuniões e oficinas sobre o projeto de pesquisa com instituições parceiras, instituições socioeducativas, centros de referência acolhedores de adolescentes em situação de rua, movimentos sociais representantes da população em situação de rua e demais atores sociais.

O tamanho das redes de cada território foi estimado a partir de contato com as sementes e do seguinte questionamento "quantas pessoas você conhece neste território que possuam idades 


\section{Revista \\ Latina \\ de Sociologia}

Revista Latina de Sociología (RELASO)

Vol. 9, núm. 2 (2019), pp. 59-76

ISSN-e 2253-6469

DOI: https://doi.org/10.17979/relaso.2019.9.2.6827

próximas as suas e exercem atividades semelhantes em situação de rua“? Foram definidos 10 territórios e/ou regiões da cidade, melhor descritos na figura 2, cuja vulnerabilidade infanto-juvenil e a exposição a riscos são características marcantes do espaço. Os territórios foram posteriormente agrupados em três tipologias de adolescentes em situação de rua encontrados, em adaptação às ideias propostas por Martins (1996).

Os sujeitos da pesquisa foram 86 adolescentes em situação de rua, com faixa etária entre 12 a 19 anos e a seleção dos participantes levou em consideração os critérios de aplicação do RDS.

Simultâneo a aplicação da técnica, foi utilizado o método TLS - Time-Location Sampling (amostragem espaço-tempo), a fim de considerar os horários, locais e características de cada espaço da rua pesquisado e sua relação com as vulnerabilidades ao uso de drogas especificamente (Veloso Filho, 2013).

Na implementação do RDS desenvolvemos uma sistematização de trabalho com os elementos essenciais que compõem a abordagem RDS, na coleta de dados, nos seus limites e potencialidades, considerando informações destacadas em diário de campo.

Brignol, Amorim e Dourado (2016) em estudo sobre os fatores de risco sociodemográficos, biológicos e de comportamentos sexuais associados à infecção por HIV, na cidade de Salvador entre homens que fazem sexo com homens, revelou que pesquisas utilizando esta técnica são adequadas para estudos com populações de difícil acesso, a medida que produzem resultados mais robustos e permite acesso a perfis demográficos semelhantes dos participantes em comparação ao encontrado em outras pesquisas de RDS.

Heckathorn (1997) afirma que o RDS oferece um sistema de incentivo duplo, ao qual chamou “de recompensa ou ressarcimento", que busca aliar o poder da influência social do entrevistado sobre sua rede social, amigos ou colegas que constituem a rede de relação em determinado contexto/território, às oportunidades de uma gratificação pessoal, ou recompensa, conforme etapa definida.

Procura-se ressarcir pela colaboração com o estudo, diminuindo assim os constrangimentos ou receios em participar da pesquisa. Existe um incentivo primário para aquele indivíduo que participar da pesquisa e um incentivo secundário para aqueles indivíduos que além de participar encaminha outros pares, ou seja, novos candidatos dentro de sua rede de amigos, para a entrevista.

O RDS possui um esquema de recrutamento baseado na identificação de "sementes". Denomina-se sementes aqueles sujeitos que, dentro da população-alvo ou rede social a ser pesquisada, possui uma expressiva influência sobre o grupo e é respeitado e valorizado pelos seus membros. No processo de coleta de dados, as sementes são os pontos chave para acesso e infiltração na rede. Elas devem concordar com a proposta de pesquisa e possuir a motivação necessária para recrutar pares semelhantes a ela que possam responder o questionário (Nastad, 2014).

Em nossa pesquisa, foi elaborada um sistema de incentivo a fim de ressarcir o adolescente em situação de rua em função do tempo gasto na participação da pesquisa, estabelecendo assim um benefício que ao mesmo tempo fosse simbólico e material, de acordo com as necessidades de cada participante. Assim, conforme discussão com parceiros e realização de mapeamento, observou-se que em sua maioria os adolescentes desenvolviam algum tipo de atividade lucrativa na rua. A partir disso, 


\begin{tabular}{|l|l|l|}
\hline Revista & & $\begin{array}{l}\text { Revista Latina de Sociología (RELASO) } \\
\text { Vol. 9, núm. 2 (2019), pp. 59-76 } \\
\text { ISSN-e 2253-6469 } \\
\text { DoI: https://doi.org/10.17979/relaso.2019.9.2.6827 }\end{array}$ \\
de Sociologia & &
\end{tabular}

foi estabelecido um perfil de gratificação onde a equipe de pesquisadores buscava adquirir/comprar os produtos que os adolescentes vendiam na rua como forma de sustento, retribuindo-os, desta forma, pelo tempo gasto na pesquisa. Ressaltasse que a gratificação recebida pelos participantes está inclusa dentro da metodologia como forma de acessar as populações de difícil acesso, já foi utilizada por outros pesquisadores (Baptista, 2016; Santos, 2013) e foi aprovado pelo Comitê de Ética da Universidade Estadual de Feira de Santana (UEFS), tendo sido aprovada sob Parecer 2.027.689, respeitando os termos da Resolução 466/12, do Conselho Nacional de Saúde (CNS).

\section{Tipologia do grupo pesquisado e análise dos dados}

Em adaptação às ideias de Martins (1996) que, em censo com crianças e adolescentes em situação de rua na cidade de São José do Rio Preto - SP, que definiu seis tipologias de adolescentes em situação de rua, essa pesquisa adaptou três tipologias fundamentadas na experiência de coleta de dados, nos 10 territórios pesquisados (figura 2), onde encontramos 86 adolescentes em situação de rua.

Fig. 2. Distribuição dos territórios e reconhecimento das tipologias encontradas



LEGENDA:

RODOVIÁRIA

E PRAÇA

Adolescentes em situação de rua, tipo 1.

FEIRA LIVRE Adolescentes em situação de rua, tipo 2.

SINALEIRA Adolescentes em situação de rua, tipo 3.

Dados da pesquisa de campo em adaptação as tipologias de Martins, 1996. 


\begin{tabular}{|l|l|l|}
\hline Revista & & $\begin{array}{l}\text { Revista Latina de Sociología (RELASO) } \\
\text { Vol. 9, núm. 2 (2019), pp. 59-76 } \\
\text { ISSN-e 2253-6469 } \\
\text { DoI: https://doi.org/10.17979/relaso.2019.9.2.6827 }\end{array}$ \\
de Sociologia & &
\end{tabular}

Fonte: Elaboração dos autores

Tipo 1 - Adolescentes que buscam renda para família e/ou para seu próprio sustento (trabalho de caráter diurno), alimentam-se nas ruas, costumam chegar tarde da noite em casa, para dormir e têm lazer no bairro onde moram. Vínculo Familiar Instável.

Tipo 2 - Adolescentes que buscam renda para família e/ou para seu próprio sustento (trabalho durante o dia, nas feiras livres), acompanhados de familiares e/ou outras pessoas, alimentam-se e dormem em casa, mas têm lazer constante nas ruas. Vínculo familiar estável.

Tipo 3 - Adolescentes que buscam renda para seu próprio sustento (atividades diversas nas principais sinaleiras), alimentam-se e dormem em casa e migram, constantemente, de atividades, espaços geográficos e horários que exercem suas funções, em virtude de conflitos familiares, disputas por territórios ou diminuição do lucro e têm lazer nos espaços públicos ou nas ruas. Vínculo Familiar instável.

Por fim ressalta-se que a análise e tratamento dos dados neste trabalho foi realizado considerando o conjunto de instrumentos e técnicas utilizadas e informações obtidas por meio do diário de campo, mapeamento territorial, questionário sociodemográfico, conhecimento das redes sociais no território e roda de conversa entre instituições parceiras, utilizando de analises documentais, leituras flutuantes e conteúdo das entrevistas. Foram selecionadas as informações mais relevantes e que apresentavam semelhanças com o problema de pesquisa, tais dados foram descritos conforme cada etapa da pesquisa e ilustrado por meio de quadros comparativo que se encontra nos resultados e discussão deste trabalho. A técnica de análise dos dados adotada foi a leitura flutuante.

Como critério de inclusão e exclusão destaca-se que os sujeitos da pesquisa foram adolescentes, em situação de rua, com faixa etária entre 12 a 19 anos, considerando critério da Organização Mundial da Saúde (OMS) e estudos semelhantes, que vivem ou trabalham no ambiente da rua e cujo contexto principal de suas relações, aprendizagens e afetos são mais estabelecidos na rua, ou seja, passam a maior parte do dia na rua e retornam para casa à noite. Foram excluídos da coleta dos dados adolescentes com distúrbios mentais ou psíquicos evidentes, com comprometimento cognitivo, auditivo ou que possuíam qualquer outra incapacidade de responder às questões.

Não houve divisão de categorias temáticas para analise em função do foco deste trabalho ter sido exclusivamente avaliar as questões metodológicas de aplicação da técnica RDS em população de adolescentes em situação de rua, utilizando-se para isso do relato de experiência dos pesquisadores, instrumentos utilizados (mapeamento territorial, diário de campo) e avaliação da adesão pelos entrevistados (preenchimento dos questionário de cada território pesquisado).

\section{Resultados e Discussão}

Foram realizadas para a amostragem em cadeia dos adolescentes, sete ondas de pesquisa/recrutamento durante o dia junto às duas tipologias com maior número de adolescentes em situação de rua (tipologias 2 e 3). Os territórios foram as sinaleiras e feiras livres (Figura 2) que são 


\begin{tabular}{|l|l|l|}
\hline Revista & & $\begin{array}{l}\text { Revista Latina de Sociología (RELASO) } \\
\text { Vol. 9, núm. 2 (2019), pp. 59-76 } \\
\text { ISSN-e 2253-6469 } \\
\text { DoI: https:// doi.org/10.17979/relaso.2019.9.2.6827 }\end{array}$ \\
de Sociologia & &
\end{tabular}

espaços de renda dos adolescentes e seus familiares, sejam vendendo frutas e verduras ou lavando carros.

Três ondas de recrutamento da pesquisa foram realizadas com coleta de dados em pontos críticos como a rodoviária e logradouros de praças públicas 1 e 2 (Figura 2), espaços de maior concentração de atividades de exploração sexual, comercial e de maior uso de drogas, tendo maior concentração dos adolescentes em situação de rua da tipologia 1.

O RDS postula que os membros de uma rede social, sejam elas de usuários de drogas injetáveis ou profissionais do sexo, tem maior poder de infiltração, influência social e convencimento dos seus membros do que qualquer outro sujeito, o RDS torna assim o entrevistado àquele que recruta novos candidatos a entrevista (Herckathorn, 1997).

Por outro lado, os territórios habitados por pessoas em situação de rua são construídos por um conjunto de regras e demarcados por valores coletivos e individuais que exercem uma função singular nas disputas sociais, na expressão dos modos de vida e no reconhecimento de variadas identidades no ambiente da rua, sejam elas de crianças, adolescentes ou adultos (Macerata, Soares \& Ramos, 2014).

Na coleta de dados, particularmente nas feiras livres e sinaleiras (tipologia 2 e 3), percebeuse que os sujeitos inseridos em tais ambientes eram adolescentes com práticas de trabalho diferenciadas, mas em contexto de vulnerabilidade e risco no qual houve um reconhecimento mútuo da rua enquanto lugar de pertencimento e onde as relações são mediadas por estratégias de autoproteção e automanutenção contra as ameaças impostas pelo meio.

Foi verificado que os adolescentes (tipologia 2 e 3) passam maior parte do tempo nesses ambientes, geralmente chegam entre cinco e sete horas da manhã e retornam apenas no fim da tarde, relatam que vão para as ruas a procura de sustento para si e para sua família, e verifica-se que em sua maioria são acompanhados/chefiados por um adulto.

Nas feiras livres, tem como responsáveis os donos das barracas, seus pais ou um parente próximo, que em alguns casos estão vendendo em um ponto e os deixam em outro e, nas sinaleiras, andam frequentemente com grupos de amigos ou sozinhos e se sentem mais livres quanto aos horários de início e término das atividades. Nesse contexto, observou-se entre os grupos de adolescentes a existência significativa de um maior trânsito entre a família e a rua, sendo os vínculos parentais e com a escola caracteristicamente superficiais ou transitório e o grau de afinidade influenciado pelo tempo que passa na rua.

Nos pontos de maior risco ao uso de drogas e prostituição durante a noite (tipologia1), os adolescentes dormem, sobrevivem e compartilham estilos de vida variados, ficam até as madrugadas vendendo balas na rodoviária, dormem nas praças de alimentação ou perambula pelos pontos de prostituição onde situam a maioria das casas noturna. Nesse novo contexto a rua ganha vida, ganha sentido, ganha cor não pelo retorno financeiro promovido, mas como ambiente de liberdade para viver da forma que achar possível, mais adequada ou que preencha as lacunas das relações familiares.

Matias (2011) pondera que tais características são comuns na (re)elaboração da pessoa em situação de rua e numa maior visibilidade da existência desses sujeitos que, no espaço da rua, nas encruzilhadas, nos becos e nos muros constituem, independentemente, seus campos de interação 


\begin{tabular}{|l|l|l|}
\hline Revista & & $\begin{array}{l}\text { Revista Latina de Sociología (RELASO) } \\
\text { Vol. 9, núm. 2 (2019), pp. 59-76 } \\
\text { ISSN-e 2253-6469 } \\
\text { DoI: https://doi.org/10.17979/relaso.2019.9.2.6827 }\end{array}$ \\
de Sociologia & &
\end{tabular}

interpessoal, suas práticas sociais ou práticas de espaço diferenciadas em função do lugar onde se encontram e, acima de tudo, onde formam sua identidade e se reconhecem como sujeitos.

O trabalho de campo revelou que nesse espaço há um maior distanciamento da família e aproximação com a rua, dessa forma na rua os adolescentes se fazem, se constroem e se percebem enquanto sujeitos invisíveis para o mundo e reais para si mesmo; as experiências de agressão e violência são fatos concretos da realidade vivida e a desconfiança se torna fenômeno associado às relações de amizade na rua, pois há uma necessidade de lutar pela sobrevivência diária Macerata, Soares e Ramos (2014) reafirmam que a rua, enquanto território da existência, possui uma lógica de constante mudança reconhecida pelos sujeitos que integram seu universo, é um território de vida estranho aos costumes e moldes da sociedade moderna.

A coleta de dados realizada por meio da técnica RDS revelou múltiplas faces da adolescência em situação de rua; foram encontrados adolescentes em situação de rua que desenvolviam atividades em feiras livres, adolescentes em situação de rua que desenvolviam atividades em sinaleiras e adolescentes em situação de rua que trabalhavam a noite toda ou de modo diurno seja como profissionais do sexo ou vendedores de balas e outros alimentos; embora fossem todos adolescentes em situação de rua, suas práticas de vida eram heterogêneas, estavam inseridos numa mesma rede social, mas com graus diferenciados de risco e vulnerabilidade.

A identificação das sementes, que eram os principais sujeitos que conduziam o processo de entrevista realizado nas tipologias 2 e 3 (ambiente das feiras livres e nas sinaleiras), pesquisa durante o dia, apresentou menor dificuldade do que junto a tipologia 1, em função que neste turno as relações eram sempre mediada pela presença de um adulto, parceiro ou aliciador, estabelecendo barreiras de acesso aos adolescentes em situação de rua e reconhecendo, imediatamente, nos pesquisadores, uma ameaça ao tipo de trabalho desenvolvido pelos adolescentes.

Os limites e possibilidades do uso do RDS na pesquisa com adolescentes em situação de rua foram sumarizados no quadro 1.

Quadro 1. Compreensão das etapas em seus limites e possibilidades 


\begin{tabular}{|l|l|l|}
\hline Revista & & $\begin{array}{l}\text { Revista Latina de Sociología (RELASO) } \\
\text { Vol. 9, núm. 2 (2019), pp. 59-76 } \\
\text { ISSN-e 2253-6469 } \\
\text { DoI: https:// doi.org/10.17979/relaso.2019.9.2.6827 }\end{array}$ \\
de Sociologia & &
\end{tabular}

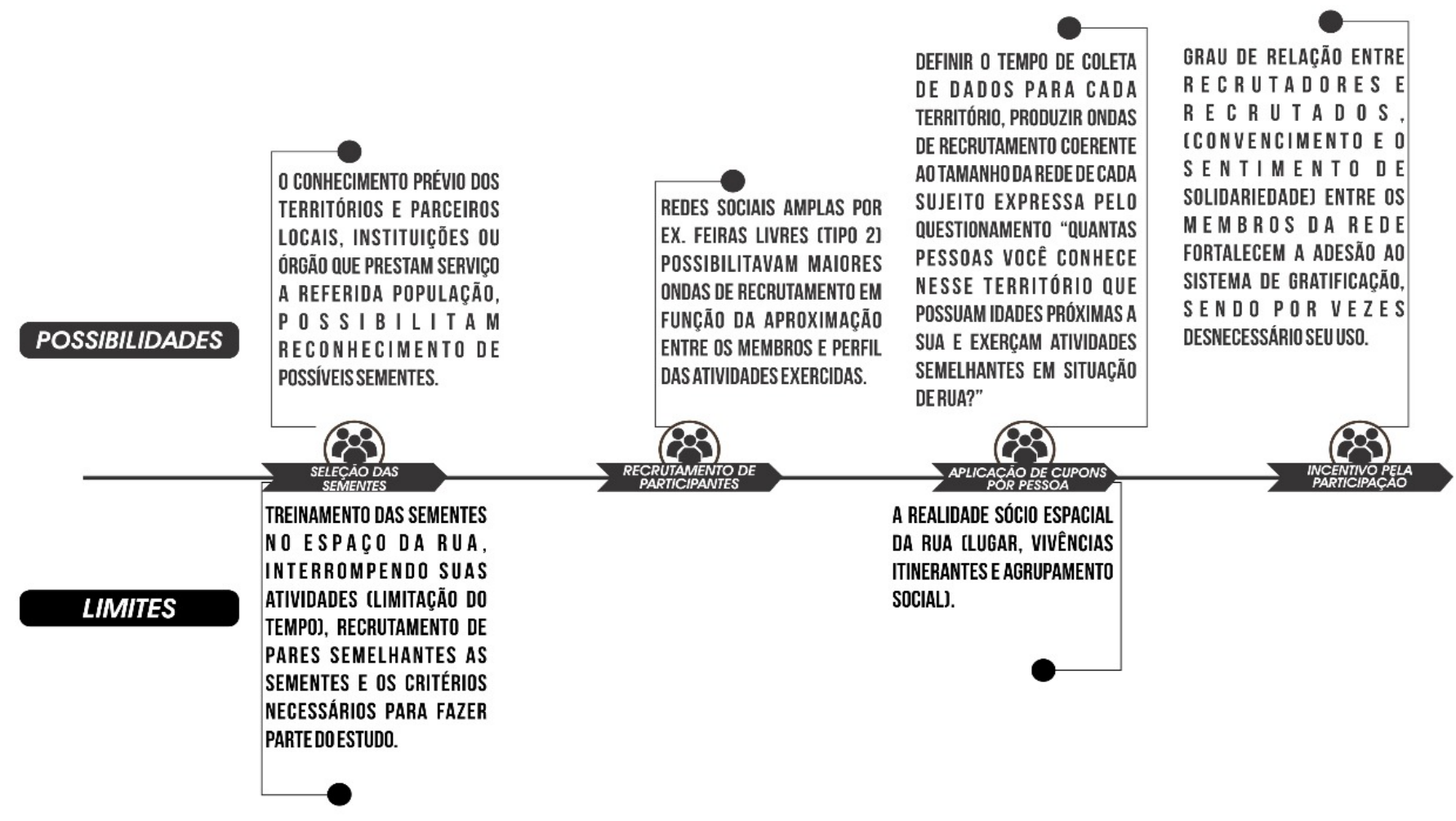

Fonte: Elaboração dos autores

Ressalta-se que a identificação das sementes deve fazer parte de uma longa avaliação que considere principalmente a quantidade de participantes a ser recrutados e a heterogeneidade da população pois através disso se permite maior acesso as redes sociais e diminuição de peculiaridades que poderiam enviesar os dados. A estruturação dessa etapa permitiu uma amostra diversificada e para isso importa que quatro elementos sejam destacados: fazer a documentação de recrutador e participantes recrutados, que recrutador e recrutado se conheçam; recrutamento por etapas para que um único sujeito não recrute todos os outros e, por fim, questionar a cada participante o tamanho da rede que ele está inserido (Nastad, 2014).

O turno da noite com adolescentes (tipologia 1) foi aquele que se verificou com maior dificuldade no recrutamento dos pares e geração de novas ondas, talvez em função do tamanho reduzido das redes sociais de cada semente e do distanciamento entre seus membros. A maioria dos adolescentes encontrados eram maior idade e estavam distribuídos em territórios onde o vínculo entre as pessoas caracterizava-se como distantes e/ou instáveis contribuindo para maior dificuldade de adesão a técnica e onde o sentimento de insegurança dos participantes foi uma realidade mais concreta do que em outros espaços. 


\section{Revista \\ Latina \\ de Sociologia}

Revista Latina de Sociología (RELASO)

Vol. 9, núm. 2 (2019), pp. 59-76

ISSN-e 2253-6469

DOI: https://doi.org/10.17979/relaso.2019.9.2.6827

Quanto ao recrutamento dos pares, nas ondas de recrutamento realizada durante o dia (tipologia 2 e 3) obteve-se maior retorno, êxito e participação dos adolescentes em situação de rua; fato que pode estar relacionado à dinâmica de vida nos territórios caracterizada por maior abertura social e liberdade de locomoção entre os espaços, diferente do período da noite, o qual demanda restrições absolutamente necessárias a manutenção de suas vidas no espaço da rua.

O período noturno (tipologia 1), apresentou maior limitação na identificação das sementes e na relação entre pesquisadores e entrevistados.

A aplicação de fichas/cupom de indicação, definindo um número RDS de cada participante, não foi realizada devido o local de coleta dos dados pois as entrevistas ocorreram com adolescentes no espaço da rua onde exerciam suas atividades; concomitante a isso as vivências itinerantes dos adolescentes (não estar todos os dias no mesmo local, horário e tempo que ficavam na rua) impossibilitavam a distribuição de fichas.

A dificuldade de cumprimento desta etapa pode ser entendida sob a ideia que os adolescentes em situação de rua, junto as formas singulares de estar na rua e a condição social, apresentam um sentido diverso ao integrar populações de difícil acesso, pois integram uma perspectiva de baixa visibilidade social e segregação no espaço geográfico que contribuem para menor mensuração de suas características e processos grupais (Baptista, 2016).

Heckathorn (1997) afirma que o contato e/ou as conexões entre os membros e a estrutura dos vínculos sociais estabelecidos pela população-alvo é fenômeno de importância particular para efetivação do método RDS, sendo necessário aferir, sempre que possível, o modo de funcionamento e as características do grupo como algo diretamente relacionado à adesão da população.

O sistema de incentivo/gratificação ou ressarcimento estabelecido em nossa metodologia apresentou maior eficácia no trabalho junto às tipologias 2 (adolescentes das feiras livres), sendo por vezes até mesmo desnecessário seu uso; dentre todas as tipologias a que mais apresentou resistência na adequação e aceitação foi a tipologia 3 (adolescentes das sinaleiras), situação minimizada pelo grau de relação que os recrutadores tinham com os recrutados somado ao poder de convencimento sobre sua rede.

Santos (2013), em estudo com usuários de drogas vulneráveis ao HIV, pode perceber que a distribuição espacial constitui um importante marcador ao utilizar o RDS. À medida em que se realiza as entrevistas em espaços diferenciados, aumenta as possibilidades de um recrutamento homogêneo adequado à realidade da população.

Adolescentes da tipologia 2 foram nos quais as entrevistas transcorreram com maior naturalidade e onde houve maior colaboração dos participantes, sendo em muitos momentos desnecessário o incentivo em função do sentimento de solidariedade existente entre os membros da rede. Para a tipologia 1, em que foram encontrados adolescentes em situação de maior vulnerabilidade perambulando e alguns acompanhados com adultos, o processo de ressarcimento se mostrou inadequado.

Herckathorn (1997) entende que as reações ao sistema de gratificação podem variar bastante e em certo sentido podem até ser ineficazes, por exemplo, um incentivo material que não corresponda à necessidade do sujeito pesquisado em determinado momento. Um fato que pode auxiliar na 


\begin{tabular}{|l|l|l|}
\hline Revista & & $\begin{array}{l}\text { Revista Latina de Sociología (RELASO) } \\
\text { Vol. 9, núm. 2 (2019), pp. 59-76 } \\
\text { ISSN-e 2253-6469 } \\
\text { DoI: https:// doi.org/10.17979/relaso.2019.9.2.6827 }\end{array}$ \\
de Sociologia & &
\end{tabular}

condução dessa questão é o tipo de motivação que os pares vão desenvolver para com os demais, a qual pode comunicar necessidades materiais ou simbólicas.

Damacena, Szwarcwald e Barbosa Junior (2011) compreendem que nas redes sociais a solidariedade pode assumir uma função ímpar na condução das entrevistas pelos participantes, sendo que esta pode despertar o interesse pela livre participação, independentemente de qualquer tipo de gratificação envolvida.

\section{Conclusões}

A técnica RDS no referido trabalho com adolescentes em situação de rua possibilitou o reconhecimento da pluralidade de modos de vida compartilhados no espaço da rua por adolescentes em processo de risco e vulnerabilidade. Por meio da aplicação dessa abordagem metodológica, foi possível mensurar se a técnica conferiu ou não representatividade geográfica aos dados coletados nos territórios estudados.

O ambiente das feiras livres e da sinaleira (tipologias 2 e 3) durante o dia revelou ser mais aberto e acessível à técnica de RDS do que o período noturno (tipologia 1), momento de maior limitação na identificação das sementes e na relação entre pesquisadores e entrevistados.

A experiência de campo revelou que a utilização do RDS, enquanto técnica de coleta de dados, se alinha ao processo dinâmico das redes sociais no espaço da rua, mas ao mesmo tempo desperta questionamento quanto ao rigor sistemático das etapas do método, em populações com culturas de relações diversas com o espaço.

Assim as dificuldades na aplicação da técnica de RDS nessa pesquisa foram: a identificação de algumas sementes e a aplicação do sistema de cupons, em função das características dos territórios pesquisados e vivências itinerantes dos adolescentes em situação de rua que num dia, estavam em um ponto e em outro dia, estavam em outro. Tal situação tornou difícil e questionável tanto o recrutamento dos pares quanto o retorno dos adolescentes, sendo assim ponto controverso na coleta de dados.

O uso da técnica demonstrou-se apropriado no acesso à população, no entanto, em função da dinâmica social e características socioespaciais da realidade dos adolescentes em situação de rua, os quais possuem uma mobilidade ativa no território, a replicação desta técnica com a mesma população em espaço similar pode apresentar resultado diferente em função da variabilidade do perfil social do grupo pesquisado.

Em resposta as questões em aberto e limitações percebidas no estudo pesquisas subsequentes estão sendo realizadas, como forma de continuidade do trabalho e com o intuito de aprofundar questões relacionadas a vida na rua, valores sociais de tais sujeitos e demais comportamentos de risco.

Concomitante a isso sugere-se que estudos futuros priorize a aplicação da técnica em outros seguimentos da população em situação de rua ainda pouco explorados, tais como, crianças em situação de rua, adolescentes do sexo feminino e comunidades homoafetivas em situação de rua. 


\begin{tabular}{|l|l|l|}
\hline Revista & & $\begin{array}{l}\text { Revista Latina de Sociología (RELASO) } \\
\text { Vol. 9, núm. 2 (2019), pp. 59-76 } \\
\text { ISSN-e 2253-6469 } \\
\text { DoI: https://doi.org/10.17979/relaso.2019.9.2.6827 }\end{array}$ \\
de Sociologia & &
\end{tabular}

\section{Referências}

Adorno, R. C. F (2011). Crianças, Adolescentes e Jovens: exclusão, Vulnerabilidade, Cidadania e Modos de Vida: uma aproximação aos circuitos de trânsito pelas ruas. In: Silva, E. A., \& Micheli, D. (Org.). Adolescência uso e abuso de drogas: uma visão integrativa (pp. 303 - 314). São Paulo: Editora FAP-UNIFESP.

Baptista, J.C. (2016). Prevalência de HIV e de sífilis e seus fatores de risco em pessoas que usam substâncias psicoativas em cidades brasileiras. (Tese de Doutorado). Instituto de Saúde Coletiva, Universidade Federal da Bahia, Salvador, BA, Brasil.

Bittencourt, A. L. P.; França, L.G.; \& Goldim, J.R. (2015). Adolescência vulnerável: fatores biopsicossociais relacionados ao uso de drogas. Revista Bioética, 23(2), 311-319. https://dx.doi.org/10.1590/1983-80422015232070

Brasil. (2009). Rua: aprendendo a contar: Pesquisa Nacional sobre a População em Situação de Rua. Brasília: Ministério do Desenvolvimento Social e Combate à Fome.

Brasil. (2019). População em Situação de Rua no Brasil: o que os dados revelam? Brasília: Ministério da Cidadania.

Brignol, S., Kerr, L., Amorim, L. D., \& Dourado, I. (2016). Fatores associados a infecção por HIV numa amostra respondent-driven sampling de homens que fazem sexo com homens, Salvador. Revista Brasileira de Epidemiologia, 19(2), 256-271.

Carvalho, R.C; Nery, G. S.; Souza, S. L.; Melo, L. G.; Silva, L. C. L.; Santos, J. E. F. (2019). Adolescentes em situação de rua e uso do respondent driven sampling (rds): limites e possibilidades. Anais: Atas CIAIQ 2019 Investigação Qualitativa em Ciências Sociais, v. 3. p. 387-395.

Damacena, G. N., Szwarcwald, C. L., \& Barbosa- Junior, A. (2011). Implementation of respondentdriven sampling among female sex workers in Brazil, 2009. Cadernos de Saúde Pública (ENSP. Impresso), v. 27, p. S45-55.

Escorel, S. (2009). A saúde das pessoas em situação de rua. In BRASIL. Ministério do Desenvolvimento Social e Combate a Fome. (Org.). Rua - aprendendo a contar: Pesquisa Nacional sobre a População em Situação de Rua. Distrito Federal: Secretaria de Avaliação e Gestão da Informação, Secretaria Nacional de Assistência Social.

Heckathorn, D. (1997). Respondent-driven sampling: a new approach to the study of hidden populations. Social Problem, 44(2), 174-199.

Kendall, C. (2006). Respondent Respondent-Driven Sampling (Amostragem Conduzida pelo Entrevistado) (Apresentação de Power Point). New Orleans, LA, EUA: FIOCRUZ.

Koller, S. H., \& Hutz, C. S. (1997). Questões sobre o desenvolvimento de crianças em situação de rua. Estud. psicol, 2(1), 175-197.

Kunz, G.S., Heckert, A.L., \& Carvalho, S.V. (2014). Modos de vida da população em situação de rua: inventando táticas nas ruas de Vitória/ES. Fractal: Revista de Psicologia, 26(3), 919-942. 


\begin{tabular}{|l|l|l|}
\hline Revista & & $\begin{array}{l}\text { Revista Latina de Sociología (RELASO) } \\
\text { Vol. 9, núm. 2 (2019), pp. 59-76 } \\
\text { ISSN-e 2253-6469 } \\
\text { DoI: https:// doi.org/10.17979/relaso.2019.9.2.6827 }\end{array}$ \\
de Sociologia & &
\end{tabular}

Macerata, I.; Soares, J. G. N.; Ramos, J.F.C. (2014). Apoio como cuidado de territórios existenciais: Atenção Básica e a rua. Interface (Botucatu), Botucatu, v. 18, supl. 1, p. 919-930.

Martins, R. A. A. (1996). Criança e o Adolescente em Situação de Rua: Definições, Evolução e Políticas de Atendimento. Coletâneas da Anpepp, 1(12), 35-44.

Matias, H.J.D. (2011). Jovens em situação de rua: espaço, tempo, negociações de sentido. Psicologia \& Sociedade, 23(2), 237-247.

Mello, S.L. (2001). A violência Urbana e a Exclusão dos Jovens. In: SAWAIA, Bader. As artimanhas da exclusão: analise psicossocial e ética da desigualdade social. Petrópolis: Editora Vozes, 2ed.

Moura, Y. G., Silva, E. A., \& Noto, A. R. (2009). Redes sociais no contexto de uso de drogas entre crianças e adolescentes em situação de rua. Psicol. pesq., 3(1), 31-46.

Morell, M.G.G, Silva, N.G., Lacerda, G.M. \& Araújo, N. (2010). A efetividade do uso da metodologia respondent driven sampling para vigilância comportamental do HIV em trabalhadoras do sexo na cidade de Santos. In: XVII Encontro Nacional de Estudos Populacionais. Anais eletrônicos: MGBrasil, de 20 a 24 de setembro.

Nascimento, A. (2009). Uso de solventes por crianças e adolescentes em situação de rua no Distrito Federal. (Dissertação de Mestrado) Faculdade de Ciências da Saúde, Universidade de Brasília. Recuperado de http://repositorio.unb.br/handle/10482/3868

NASTAD. National Alliance of State \& Territorial AIDS Directors (2014). Respondent-Driven Sampling: A Resource Guide for Steering Committee Members. EUA: NASTAD.

Neiva-Silva, L., \& Koller, S. H. (2002). A rua como contexto de desenvolvimento. In Lordelo, E. R., Carvalho, A. M. A., \& Koller S. H. (Orgs.). Infância brasileira e contextos de desenvolvimento. (pp. 205-230). São Paulo: Casa do Psicólogo.

Nery, G. S. (2018). Fatores de risco ao uso e abuso de substâncias psicoativas em adolescentes em situação de rua na cidade de Feira de Santana-Bahia. 82f. Dissertação de Mestrado. Universidade Estadual de Feira de Santana, Bahia.

Pinho, A. (2010). Pesquisa RDS em homens que fazem sexo com homens. In. Seminário estudos e pesquisas em DST/HIV/AIDS: determinantes epidemiológicos e sociocomportamentais. Anais: ABIA, Rio de Janeiro, RJ, Brasil.

Santos N. T. V. (2013). Vulnerabilidade e prevalência de HIV e sífilis em usuários de drogas no Recife: resultados de um estudo respondent-driven sampling. (Tese de Doutorado), Centro de Pesquisas Aggeu Magalhães, Recife, PE, Brasil.

Schenker, M. \& Minayo, M.C.S. (2005). Fatores de risco e de proteção para o uso de drogas na adolescência. Ciência \& Saúde Coletiva, 10(3), 707-717.

Schonlau, M., \& Liebau, E. (2012). Respondent-driven sampling. Stata Journal, 12(1), 72-93.

Schwonke, C.R.G.B; Fonseca, A.D; Gomes, V.L.O. (2009). Vulnerabilidades de adolescentes com vivências de rua. Rio de Janeiro, Esc. Anna Nery, v. 13, n. 4, p. 849-855, Dez. 


\begin{tabular}{|l|l|l|}
\hline Revista & & $\begin{array}{l}\text { Revista Latina de Sociología (RELASO) } \\
\text { Vol. 9, núm. 2 (2019), pp. 59-76 } \\
\text { ISSN-e 2253-6469 } \\
\text { DoI: https://doi.org/10.17979/relaso.2019.9.2.6827 }\end{array}$ \\
de Sociologia & &
\end{tabular}

Trino, A. T., Machado, M. P. M., \& Rodrigues, R.B. (2015). Conceitos norteadores do cuidado junto à população em situação de rua. In Teixeira, M., \& Fonseca, Z. (Orgs.). Saberes e Práticas na Atenção Primária à Saúde: cuidado à população em situação de rua e usuários de álcool, crack e outras drogas. (1a ed., pp. 27-44.). São Paulo: Hucitec.

Varanda, W., \& Adorno, R. C. F. (2004). Descartáveis urbanos: discutindo a complexidade da população de rua e o desafio para políticas de saúde. Saúde e Sociedade, 13(1), 56-69.

Veloso Filho, C. L. (2013). Cenas de uso de crack no município do Rio de Janeiro: perfil em 2011/2012. 2013. Tese (Epidemiologia em Saúde Pública) - Escola Nacional de Saúde Pública Sergio Arouca, Rio de Janeiro. 